\title{
Reliability of a questionnaire on sickness absence with specific attention to absence due to back pain and respiratory complaints
}

\author{
Alex Burdorf, Wendel Post, Ton Bruggeling
}

\begin{abstract}
Objective-To evaluate the performance of a questionnaire on sickness absence due to back pain and respiratory disorders with the view of using sickness absence as a measure of morbidity.

Methods-A cross sectional survey was conducted among 511 male workers aged 20-65, drawn from the personnel register of an animal feed mill. The response was $404(79 \%)$ participants. Data on sickness absence in the six months before the survey were collected by a self administered questionnaire and by sickness absence records. Correlation between both methods was examined for prevalence, duration, and frequency of overall sickness absence, back pain absence, and absence due to respiratory complaints.

Results-The questions about the prevalence of sickness absence from all causes, back pain, and respiratory complaints showed a high specificity of $91 \%, 97 \%$, and $98 \%$, respectively. The sensitivities of these questions were $79 \%, 88 \%$, and $13 \%$, respectively. The survey found a moderate agreement between the questionnaire and the medical register for duration and frequency of overall sickness absence with $\kappa$ values of 0.54 and 0.50 . A good agreement was found for back pain absence with $\kappa$ values of 0.65 and $0 \cdot 61$. Respiratory absence showed a poor agreement of 0.16 and $0 \cdot 13$.
\end{abstract}

Conclusion-In epidemiological studies questionnaires might be considered a valuable source of information on overall sickness absence or absence due to back pain which lasted for at least two weeks in the past six months. Whether a questionnaire survey is a reliable source of data on sickness absence due to respiratory complaints remains to be seen.

(Occup Environ Med 1996;53:58-62)

Keywords: sickness absence; questionnaire

Sickness absence is an important indicator of morbidity in public health and occupational medicine. Sickness absence may be used as a measure of a worker's ability to cope with normal roles at work, as a measure of use of health services, and as a measure of lost productivity. ${ }^{1}$ Although sickness absence is influenced by social and psychosocial factors as well as illness, it has been used as a measure of morbidity that assesses functional impairment in relation to work load. ${ }^{2}$ In general, sickness absence registers are to be preferred in research but for various reasons it may be (too) difficult to link individual people to records of companies or insurance boards. Moreover, company registers may be incomplete or lack validity. Hence, many epidemiological studies have relied on questionnaires to collect data on various measures of sickness absence. Not uncommonly, studies based on questionnaire data have applied sickness absence as an indicator of severity of the disease under study..$^{4-6}$

The validity of sickness absence collected by questionnaire is not well documented. One study on the occurrence of low back pain among construction workers compared a self administered questionnaire with an interview and concluded that the self reported inability to perform work during the past year agreed with only $43 \%$ of all sickness absence periods mentioned in the interview. ${ }^{7}$ Another study compared a question on absence from work due to back pain during the past 12 months with certified sickness absence of two days or more. The sensitivity of the question was $82 \%$ and the specificity was $84 \%{ }^{8}$

In the current study the reliability of a questionnaire on sickness absence was evaluated for the prevalence, frequency, and duration of periods of sickness absence due to back pain, respiratory disorders, or other causes. As part of a health survey, workers in an industrial company were asked to fill out a questionnaire on health complaints and consequent sickness absence. The reliability of these sickness absence data was assessed with the company records as reference.

\section{Methods}

The population under study consisted of 511 male workers at different production sites, maintenance services, and offices in an animal feed factory. Subjects currently employed in their job for at least 12 months were invited to participate in the study. The mean (range) age of the group was $40(20-65)$ years. The framework of the study consisted of a questionnaire survey aimed at describing the occurrence of back pain and respiratory symptoms as both were perceived as important health problems. The subjects were informed about this objective of the study and were told that the questionnaire information could be matched with their medical records. Confidential treatment of all data gathered was guaranteed. The self 
administered questionnaire was handed out and returned by freepost to the medical service on site. After three weeks reminders including a new questionnaire were sent out. Questions concerned personal information, work history, back pain and respiratory symptoms, overall sickness absence, and sickness absence specific to back pain and to respiratory complaints. A detailed account on the prevalence of respiratory complaints and its impact on sickness absence has been published elsewhere. ${ }^{9}$

This report focuses on the reliability of the questions on sickness absence due to back pain and to respiratory symptoms. The considerations for this choice were twofold. Firstly, these diseases were expected to show an ample difference in the prevalence of sickness absence with a high occurrence of back pain and a moderate occurrence of respiratory symptoms. Secondly, structured questionnaires comprising questions on the occurrence of back pain and respiratory complaints with good reliability were available, thus, offering valid self reported diagnoses. The questions on back pain were derived from a standardised Nordic questionnaire ${ }^{10}$ and the questions on respiratory complaints closely resembled those from a standardised European questionnaire on chronic non-specific lung disorder. ${ }^{11}$ Among others, additional questions on sickness absence were added to these two standardised questionnaires. Subjects were asked whether they had been absent in the six months before filling out the questionnaire. The primary question was "Have you been absent from work during the past six months because of sickness?". If the reply was positive, questions were asked about frequency of absence and total number of workdays with sickness absence. These questions were phrased "How many times have you been absent from work during the past six months because of sickness?" and "What is the total number of days that you have been absent from work during the past six months because of sickness?". The answer to the questions on duration was restricted to the three categories 1-7 days, 8-14 days, and more than 14 days. Frequency was measured on a three point scale with values $1,2-5$, and more than 5 .

Similar questions were included for sickness absence due to back pain and to respiratory symptoms, starting with primary questions on the occurrence of sickness absence.

The company sickness absence register was used to retrieve records covering the past six months. If a worker falls ill, he reports his absence to the administration office. Subsequently, these workers are visited by a trained layman who reports on complaints and possible causes of illness to the register. Every week the trained layman and the occupational physician discuss the medical background of absent workers. The company register comprises frequency and duration of sickness absences and diagnoses according to the international classification of diseases. Workers who have been absent for four weeks or those with severe problems are examined by the occupational physician who, subsequently, documents his diagnosis in the company register. For the purpose of this study, the occupational physician categorised the causes of sickness absence into six groups; respiratory symptoms, influenza, back pain, other musculoskeletal disorders, other diseases, and reasons unknown. This study focused on absence due to back pain and to respiratory symptoms underlying chronic bronchitis and asthma. These respiratory symptoms comprised complaints of chronic cough, chronic sputum secretion, wheeze, shortness of breath, and attacks of chest tightness (asthma). These complaints and consequent absences were also asked for in the questionnaire. The following outcomes of sickness absence per worker were collected: absence prevalence (at least one period of sickness absence), absence duration (total number of workdays with sickness absence), and absence frequency (total number of periods of sickness absence).

All variables of sickness absence refer to absence in the six months before the administration of the questionnaire. This choice was made rather arbitrarily to avoid strong recall bias. The recollection of circumstances surrounding the absence is likely to be worse for periods of absence in the more distant past. ${ }^{12}$

Correlation between the questionnaire and the sickness absence register was first evaluated by calculating the sensitivity and specificity of the primary questions on sickness absence, with the company register as the gold standard. The sensitivity expresses the proportion of workers with sickness absence who are correctly identified by the questionnaire. The specificity is the proportion of workers without sickness absence who are correctly identified by the questionnaire. These measures are derived from $2 \times 2$ tables and, hence, do not take into account the distribution of frequency and duration of sickness absence. Therefore, the concordance was also evaluated by the percentage agreement over all categories and by Cohen's $\kappa$ (including zero) in $4 \times 4$ tables. This measure also takes into account that judged agreement depends on both the number of agreements between categories and the number of disagreements between categories and can be regarded as a chance corrected agreement. ${ }^{1314}$ As a rule of thumb, $\kappa$ values less than 0.4 represent poor agreement, $0.4-0.6$ moderate agreement, $0.6-0.8$ substantial or good agreement, and $0 \cdot 8-1 \cdot 0$ (almost) perfect agreement. ${ }^{14}$ Confidence intervals were also found. ${ }^{13}$

\section{Results}

The initial response to the questionnaire was $81 \%$ (413 respondents). Nine questionnaires were incomplete, resulting in a study sample of 302 production workers (response $76 \%$ ) and 102 office workers (response 86\%). Analysis of the sickness absence records showed that in both groups the frequency and duration of sickness absence was slightly higher among non-responders than among responders. The percentage of workdays with sickness absence in the past six months 
(absence rate) among non-responders was $6.2 \%$ in comparison with $5.2 \%$ among responders. A substantial part of the higher sickness absence among non-responders could be explained by longer periods of absence due to influenza. No significant difference in distribution of specific sicknesses of non-responders was found between office workers and production workers.

During the six months of the survey the sickness absence rate in the total group was $5 \cdot 2 \%$ with respiratory symptoms accounting for $5.8 \%$ of the days lost, influenza $15.3 \%$, back pain $13.5 \%$, other musculoskeletal disorders $13.4 \%$, and various other causes $52.0 \%$. The office workers had a significantly lower absence rate, $1.7 \% v 6.4 \%$ for production workers, primarily the result of less frequent and shorter periods of absence due to back pain and other musculoskeletal disorders. The production workers had a higher proportion of periods of absence of over 14 days.

Tables 1 and 2 present the prevalence, duration, and frequency of sickness absence reported in the questionnaire and retrieved from the sickness absence register. The question about sickness absence in the past six months was answered affirmatively by $50 \%$ of the subjects whereas the prevalence of absence in the register was $58 \%$. The prevalence of sickness absence due to back pain was $8 \cdot 2 \%$ and to respiratory disorders $7 \cdot 9 \%$. In general, subjects underestimated the total number of workdays with sickness absence and overestimated frequencies of five or more spells of sickness absence. Striking differences were found for absence caused by respiratory symp-

Table 1 Frequency of self reported sickness absence and recorded sickness absence during six months among 404 male workers in an animal feed mill

\begin{tabular}{lrrrrlll}
\hline & \multicolumn{9}{c}{ Questionnaire } & & \\
\cline { 2 - 6 } Cause of sickness absence & Register & 0 & 1 & $2-5$ & $>5$ & & Value (95\% CI) \\
\hline All causes: & 0 & 153 & 14 & 2 & 0 & Sensitivity (\%) & $79(75-83)$ \\
& 1 & 39 & 75 & 22 & 6 & Specificity (\%) & $91(88-94)$ \\
& $2-5$ & 10 & 35 & 46 & 2 & Agreement (\%) & $68(61-76)$ \\
& $>5$ & 0 & 0 & 0 & 0 & $\kappa$ & $0.50(0 \cdot 43-0.57)$ \\
Back pain: & 0 & 360 & 8 & 3 & 0 & Sensitivity (\%) & $88(85-91)$ \\
& 1 & 4 & 16 & 5 & 2 & Specificity (\%) & $97(95-99)$ \\
& $2-5$ & 0 & 4 & 2 & 0 & Agreement (\%) & $93(89-97)$ \\
& $>5$ & 0 & 0 & 0 & 0 & $\kappa$ & $0.62(0.54-0.70)$ \\
Respiratory symptoms: & 0 & 364 & 5 & 2 & 1 & Sensitivity (\%) & $13(10-16)$ \\
& 1 & 27 & 3 & 0 & 1 & Specificity (\%) & $98(97-99)$ \\
& $2-5$ & 1 & 0 & 0 & 0 & Agreement (\%) & $91(88-94)$ \\
& $>5$ & 0 & 0 & 0 & 0 & $\kappa$ & $0 \cdot 13(0 \cdot 06-0 \cdot 20)$ \\
\hline
\end{tabular}

Table 2 Duration of self reported sickness absence and recorded sickness absence during six months among 404 male workers in an animal feed mill

\begin{tabular}{lrrrrrlll}
\hline \multirow{2}{*}{$\begin{array}{l}\text { Cause of sickness } \\
\text { absence (days) }\end{array}$} & \multicolumn{6}{c}{ Questionnaire } & & \\
\cline { 3 - 7 } & Register & 0 & $1-7$ & $8-14$ & $>14$ & & Value (95\% CI) \\
\hline All causes: & 0 & 153 & 11 & 1 & 4 & Sensitivity (\%) & $79(75-83)$ \\
& $1-7$ & 25 & 63 & 3 & 4 & Specificity (\%) & $91(88-94)$ \\
& $8-14$ & 15 & 26 & 9 & 4 & Agreement (\%) & $69(62-76)$ \\
& $>14$ & 9 & 12 & 12 & 53 & $\kappa$ & $0.54(0 \cdot 48-0 \cdot 60)$ \\
Back pain: & 0 & 360 & 7 & 2 & 2 & Sensitivity (\%) & $88(85-91)$ \\
& $1-7$ & 2 & 6 & 0 & 0 & Specificity (\%) & $97(95-99)$ \\
& $8-14$ & 1 & 1 & 2 & 1 & Agreement (\%) & $94(90-98)$ \\
& $>14$ & 1 & 3 & 3 & 13 & $\kappa$ & $0.65(0 \cdot 58-0 \cdot 72)$ \\
Respiratory symptoms: & 0 & 364 & 5 & 1 & 2 & Sensitivity (\%) & $13(10-16)$ \\
& $1-7$ & 21 & 1 & 0 & 0 & Specificity (\%) & $98(97-99)$ \\
& $8-14$ & 4 & 0 & 0 & 0 & Agreement (\%) & $91(88-94)$ \\
& $>14$ & 3 & 0 & 0 & 3 & $\kappa$ & $0 \cdot 16(0 \cdot 10-0 \cdot 22)$ \\
\hline
\end{tabular}

toms. The questionnaire underestimated the prevalence of absence from respiratory symptoms by $63 \%$ and, consequently, duration and frequency of sickness absence were also underestimated.

Both tables also show the performance of the primary questions on sickness absence due to all causes, to back pain, and to respiratory complaints. The sensitivity of these questions was $79 \%, 88 \%$, and $13 \%$, respectively. The specificity of these questions was high, varying between $91 \%$ and $97 \%$. No significant differences were found between office workers and blue collar workers. The proportional agreement was high for the prevalence, duration, and frequency of sickness absence due to back pain and to respiratory symptoms, and was substantially lower for overall sickness absence. The questionnaire was most accurate for subjects without any sickness absence and those with sickness absence of over 14 workdays. The percentage agreement was strongly influenced by negative answers. The $\kappa$ statistics indicated a substantial agreement between the questionnaire and the medical records for prevalence, duration, and frequency of absence due to back pain. The questions on overall sickness absence suggested a moderate reliability whereas the questions on absence due to respiratory symptoms showed only poor reliability. In general, the percentage agreement and $\kappa$ values were slightly higher among the blue collar workers than the office workers but no systematic differences were found.

\section{Discussion}

The purpose of this study was to validate a self administered questionnaire on sickness absence by comparing it with absence data retrieved from company medical records. The results suggest that for overall sickness absence and sickness absence due to back pain the questionnaire data on prevalence, duration, and frequency compared relatively well with the company absence records. In contrast, a large discordance was found for sickness absence due to respiratory symptoms.

Several limitations must be considered when evaluating these results. Firstly, the nonresponse to the questionnaire was about $20 \%$. Analysis of non-response showed that prevalence and duration of sickness absence was higher among non-responders than responders, both among the office workers and the production workers. This could partly be explained by differences in absence from influenza. Separate analyses for absence from back pain and respiratory symptoms showed a slightly biased response in both groups, although not significant. As the office workers and production workers showed similar patterns of non-response in relation to causes of sickness absence, it was thought appropriate to combine both groups in the analysis. The biased response may be due to the survey procedure of informing all workers of the purpose of the study including the fact that information from the questionnaire could be linked to the 
company register. Although confidentiality was guaranteed, this may have caused workers with high sickness absence to refrain from participating. It is difficult to envisage how the differential response has influenced the correlation between the questionnaire and the medical records.

Secondly, incomplete medical records amounted to $5 \cdot 7 \%$ of the total number of workdays with sickness absence in the entire population under study. Diagnostic information in the medical register was lacking for about $25 \%$ of the short spells of sickness absence. As a consequence, the questionnaire may provide information on specific diagnosis of spells of absence lasting only one or two days that will not have been recorded in the medical register of the company. Such a trend was especially present for back pain that lasted less than one week. In contrast, the number of short spells of absence due to respiratory symptoms in the questionnaire was substantially underestimated compared with the medical records.

Thirdly, differences between the sources of data may have introduced discordance. The questionnaires used are meant to survey back pain and respiratory complaints of chronic non-specific lung disorder in an epidemiological framework. Some questions on sickness absence were added. The questionnaires presented to the subjects the opportunity to assess anonymously their complaints and the consequences for temporary disability. This approach essentially differs from the assessments of the trained layman and the occupational physician who are more likely to tag a specific diagnosis to a spell of sickness absence. The influence of this conceptual difference on the comparability of both methods is difficult to predict.

Fourthly, information in the questionnaire was collected with closed ended questions in contrast to open ended queries in the medical records of the sickness absence register. The standardised set of questions may be more likely to ascertain uniform and accurate information than an unstructured interview during the diagnostic evaluation by the layman and occupational physician. ${ }^{15}$ Some evidence has been presented that diseases such as chronic bronchitis and allergies of many types are recorded somewhat haphazardly in medical records. ${ }^{15}$ In the current study this hypothesis is rather speculative as the validity of the company sickness absence register could not be evaluated. It was anticipated that the validity and consistency of the medical records were high as a layman with ample experience visited the sick workers at home and, subsequently, discussed his reports and diagnoses of sickness absence with the occupational physician each week. It remains an interesting question whether the subjects underreported sickness absence due to respiratory symptoms or whether the layman and physician coding the causes of sickness absence were strongly biased towards respiratory diseases. Causes of absence from respiratory disorders were restricted to the respiratory symptoms under- lying chronic bronchitis and asthma. Symptoms such as a severe cold, pneumonia, and sore throat often intermingle with complaints of chronic cough, sputum secretion, and shortness of breath. The responders may have thought of an ordinary disease like cold where medically trained people identified symptoms of chronic bronchitis and asthma. In this survey the contribution of chronic nonspecific lung disorders to the total sickness absence, expressed by the total number of workdays with sickness absence in this population, almost equalled that of back pain. This extraordinary finding suggests some systematic bias towards diagnosing chronic non-specific lung disorder as the respiratory absence rate is usually less. ${ }^{16}$

To evaluate the reliability of the questionnaire in relation to the company register four measures of agreement were used; specificity, sensitivity, percentage of agreement, and Cohen's $\kappa$. The highest values of specificity and percentage agreement were found when most of the questions and medical records were both negative for the specific variable of sickness absence, or in other words, the prevalence of the absence was low. The low prevalence of absence from back pain in the past six months of $8.2 \%$ in this study partly explains the high specificity of $97 \%$. This is substantially higher than the reported specificity of $84 \%$ in a similar study among 418 coal miners with a prevalence of absence from back pain in the past 12 months of $13.0 \%{ }^{8}$

In epidemiological research the sensitivity may be of greater importance. The low sensitivity and $\kappa$ values for respiratory symptoms indicate that the questionnaire will fail to identify subjects with respiratory absence. This finding is partly due to the low prevalence of respiratory symptoms in the questionnaire because sensitivity, and thus $\kappa$, decreases with lower prevalences of the disease under study. The analysis also showed that the questionnaires and medical records showed a better agreement for subjects with sickness absence over 14 workdays. Hence, severe illnesses with associated long periods of absence are likely to be reported more accurately in a questionnaire. It should be borne in mind that the variables of agreement on duration and frequency of sickness absence are derived from the subgroups that replied positively to the primary questions on occurrence of sickness absence (a nested question). Single questions combining prevalence, duration, and frequency of sickness absence by including a zero category may have a lower sensitivity and specificity.

The various measures of agreement in this survey showed different aspects of validity of the questionnaire. Sensitivity and specificity can only be calculated for dichotomous variables whereas percentage agreement and $\kappa$ can also be applied to ordinal variables such as frequency and duration of sickness absence. Both frequency and duration of sickness absence do not represent information on whether the questionnaire overestimates or underestimates the prevalence and severity of sickness absence. Similar patterns were found for 
specificity and percentage agreement, largely due to a low prevalence of absence. Also, sensitivity and $\kappa$ both pointed at the limited discriminatory power of questions on respiratory complaints resulting in sickness absence.

In conclusion, this survey found moderate to good agreement between a questionnaire and a medical register for overall sickness absence and sickness absence due to back pain. The agreement, expressed by the sensitivity and the $\kappa$ coefficient, improved for sickness absence lasting over 14 working days. In epidemiological studies questionnaires might be considered a valuable source of information on overall sickness absence or absence due to back pain that lasted for at least two weeks in the past six months. Whether a questionnaire survey is a reliable source of data on sickness absence due to respiratory complaints remains to be seen.

1 North F, Syme SL, Feeney A, Head J, Shipley MJ, Marmot MG. Explaining socioeconomic differences in sickness absence: the Whitehall II study. $B M \mathcal{F}$ 1993;306:361-6.

2 Isacsson A, Hanson BS, Janzon L, Kugelberg G. The epidemiology of sick leave in an urban population in Malmö, Sweden. Scand $\mathcal{F}$ Soc Med 1992;20:234-9.

3 Bourbonnais R, Vinet A, Vézina M, Gingras S. Certified sick leave as a non-specific morbidity indicator: a casereferent study among nurses. $\mathrm{Br} \mathcal{F}$ Ind Med 1992 49:673-8.
4 Biering-Sørensen F. A prospective study of low back pain in a general population III. Medical service-work consequence. Scand $\mathcal{F}$ Rehab Med 1983;15:89-96

5 Feyer AM, Williamson A, Mandryk J, DeSilva I, Healy S. Role of psychosocial risk factors in work-related low-back pain. Scand 7 Work Environ Health 1992;18:368-75.

6 Walters S, Britton J, Hodson ME. Demographic and socia characteristics of adults with cystic fibrosis in the United Kingdom. BMF 1993;306:549-52.

7 Holmström E, Moritz U. Low back pain-correspondence between questionnaire, interview and clinical examination. Scand F Rehab Med 1991;23:119-25.

8 Agius RM, Lloyd MH, Campbell S, Hutchison P, Seaton A, Soutar CA. Questionnaire for the identification of back pain for epidemiological purposes. Occup Environ Med 1994;51:576-60.

9 Post WK, Burdorf A, Bruggeling TG. Relations between respiratory symptoms and sickness among workers in the animal feed industry. Occup Environ Med 1994;51:440-6.

10 Kuorinka I, Jonsson B, Kilbom A, Vinterberg H, Biering Sørensen F, Andersson G, Jørgensen K. Standardised Nordic questionnaires for the analysis of musculoskeletal symptoms. Appl Ergon 1987;18:233-7.

11 Minette A. Questionnaire of the European Community for Coal and Steel on respiratory symptoms. 1987-updating of the 1962 and 1967 questionnaire for studying chronic bronchitis and emphysema. Eur Respir $\mathcal{F} 1989$; 2:165-77.

12 Biering-Sørensen F, Hilden J. Reproducibility of the history of low-back trouble. Spine 1984;9:280-6.

13 Fleiss JL. Statistical methods for rates and proportions. 2nd edn. New York: John Wiley, 1981:212-36.

14 Landis JR, Koch GG. The measurement of observer agreement for categorical data. Biometrics 1977;33:159-74

15 Linet MS, Harlow SD, McLaughlin JK, McCaffrey LD. A comparison of interview data and medical records for previous medical conditions and surgery. $\mathcal{f}$ Clin Epidemiol previous medical

16 Comstock GW, Stone RW, Tonascia JA, Johnson DH. Respiratory survey findings as predictors of disability from respiratory diseases. Am Rev Respir Dis 1981;124 367-71.

\section{Occupational and Environmental Medicine and the electronic age}

OEM has an e-mail address which is 100632.3615@compuserve.com.We welcome contact by e-mail, including letters to the editor. Some of our reviewers already send us their reports by e-mail, helping to speed up the peer review process.

We are moving towards electronic publishing and for some months now we have been asking authors to send us their revised papers on disk as well as a hard copy. I am delighted to report that nearly all our authors are managing to comply with this request; far more than for other specialist journals in the BMJ Publishing group. Oddly enough, the few authors who have not sent us a disk version of their revised papers have been almost exclusively from the United Kingdom. I would be interested in suggestions for why this might be. Perhaps United Kingdom based authors read our correspondence and instructions less assiduously? Watch for revised Instructions to Authors.

The Editor 